Gynäkologe 2016 · 49:970-972 DOI 10.1007/s00129-016-3970-6

Published online: 26 September 2016

(c) Springer-Verlag Berlin Heidelberg 2016

CrossMark
Arkadius Polasik' - Thomas W. P. Friedl' · Amelie Schramm ' Fabienne Schochter ${ }^{1}$. Jens Huober ${ }^{1}$ Brigitte Rack . Elisabeth Trapp ${ }^{2} \cdot$ Marie Tzschaschel $^{2}$.

Peter A. Fasching ${ }^{3}$. Florin-Andrei Taran ${ }^{4} \cdot$ Andreas Hartkopf $^{4}$.

Andreas Schneeweiss ${ }^{5}$. Volkmar Müller ${ }^{6} \cdot$ Bahriye Aktas $^{7} \cdot$ Klaus Pantel $^{8}$. Wolfgang Janni ${ }^{1} \cdot$ Tanja Fehm $^{9}$

'Gynecology and Obstetrics, University Hospital UIm, Ulm, Germany

${ }^{2}$ Gynecology and Obstetrics, Klinikum der Ludwig-Maximilians-Universität, Munich, Germany

${ }^{3}$ Gynecology and Obstetrics, University Hospital Erlangen, Erlangen, Germany

${ }^{4}$ Gynecology and Obstetrics, University Hospital Tübingen, Tübingen, Germany

${ }^{5}$ National Center for Tumor Diseases and Department of Gynecology and Obstetrics, University Hospital Heidelberg, Heidelberg, Germany

${ }^{6}$ Gynecology and Obstetrics, , University Hospital Hamburg-Eppendorf, Hamburg, Germany

${ }^{7}$ Gynecology and Obstetrics, University Hospital Essen, Essen, Germany

${ }^{8}$ Department of Tumor Biology, University Hospital Hamburg-Eppendorf, Hamburg, Germany

${ }^{9}$ Gynecology and Obstetrics, , Heinrich-Heine-Universität, Düsseldorf, Germany

\section{Erratum to: Current role of liquid biopsy in metastatic breast cancer and future perspectives}

Erratum to:

Gynakologe (2016)

doi: 10.1007/s00129-016-3929-7

In the online version of the original article, the information on the number of cases in the DETECT study was shown incorrectly in - Fig. 1.

Please refer to the correct figure below.

The publisher apologizes for the error.

\section{Corresponding address}

\section{A. Polasik}

Gynecology and Obstetrics, University Hospital Ulm

Prittwitzstr. 43, 89075 Ulm, Germany

arkadius.polasik@uniklinik-ulm.de 


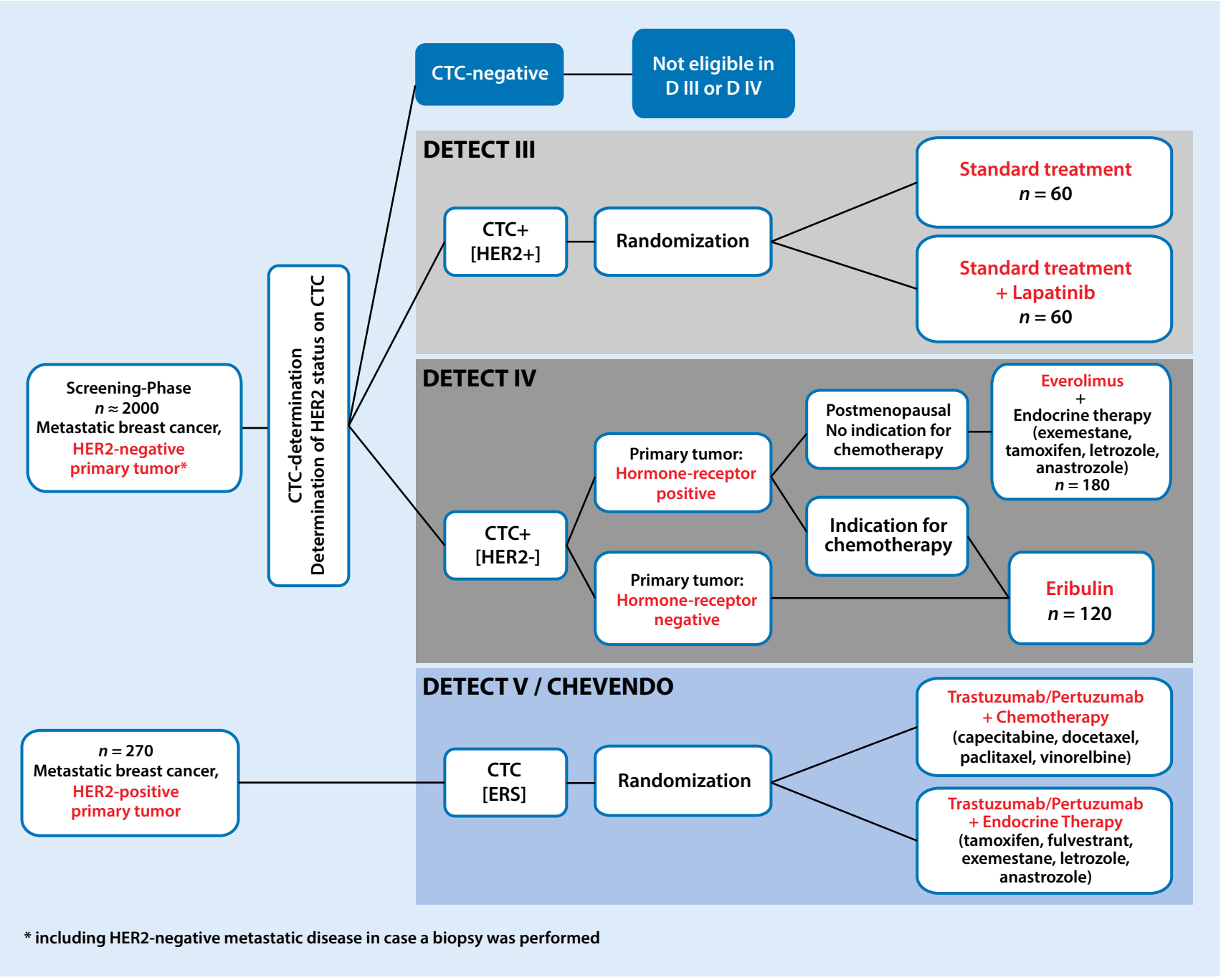

Fig. $1 \Delta$ The DETECT study concept. HER2 human epidermal growth factor receptor 2, CTC circulating tumor cells, ERS Endocrine Responsiveness Score 
Hier steht eine Anzeige.

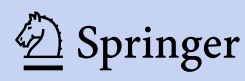

